\title{
Short-Term Investment Risk Measurement Using VaR and CVaR
}

\author{
Virgilijus Sakalauskas and Dalia Kriksciuniene \\ Department of Informatics, Vilnius University, \\ Muitines 8, 44280 Kaunas, Lithuania \\ \{virgilijus.sakalauskas, dalia.kriksciuniene\} @vukhf.lt
}

\begin{abstract}
The article studies the short-term investment risk in currency market. We present the econometric model for measuring the market risk using Value at Risk $(V a R)$ and conditional $\operatorname{VaR}(C V a R)$. Our main goals are to examine the risk of hourly time intervals and propose to use seasonal decomposition for calculation of the corresponding $\mathrm{VaR}$ and $C V a R$ values. The suggested method is tested using empirical data with long position EUR/USD exchange hourly rate.
\end{abstract}

\section{Introduction}

Trading in the stock and currency markets has many common features, yet these markets have major differences as well. Currency market has higher volatility, which causes higher risks of trade. There are many reasons which cause substantial volatility of the currency market.

- The transactions, related to the pairs of currencies exchanged, have much more trading partners, comparing to the stock trading.

- Currency exchange attracts much more instant, even unqualified traders, while stocks' trading requires at least basic minimal financial knowledge.

- The rearrangement of stock portfolio is related to quite big taxes, comparing to relatively liberate tax policy in currency trading.

The traditional way of risk estimation in the stock markets is based on periodic risk evaluations on daily basis or even by taking longer periods. This practice is in most cases based on empirical experience and is convenient for application in trading stocks. Yet even most simple analysis of currency markets indicates, that this kind of risk evaluation could be not sufficient, as during the period of 24 hours it changes several times: for particular hours it can differ even up to four times, as it is further shown in this article.

The paper aims at the estimation of the market risk for the short-term investments in currency market by suggesting the modified RiskMetrics model, based on risk evaluation according to hourly profit alterations of the financial instrument.The second part of the article describes and evaluates the theoretical settings for risk analysis in the currency markets by applying traditional models. The econometric description and substantiation of the suggested model is presented in part 3. The fourth part presents experimental verification of the method using FOREX historical data of EUR/USD hourly exchange rate fluctuations. 


\section{Theoretical Assumptions and Notations}

One of the most widely used factors for market risk measurement is Value at Risk $(V a R)$, which is extensively discussed in scientific literature starting already from the 1990. Historically, the concept of the Value-at-Risk is related to the covariance calculation method that was first adopted by the J.P.Morgan Bank as a branch standard, called RiskMetrics model [15]. The VaR measure means the biggest loss of investment $\mathrm{R}$ during the time interval, at the fixed rate of probability $p$ of unfavorable event:

$$
P(R>V a R) \leq p,
$$

where $p$ in most cases is selected as 0.01 or 0.05 (or $1 \%$ or $5 \%$ ). The loss of investment $R$ is understood as negative difference of the buying price $P_{0}$ and the selling price $P_{1}: R=-\left(P_{1}-P_{0}\right)$. In the article profitability is denoted as $P_{1}-P_{0}$.

The VaR measurement is very popular for its relative simplicity of interpretation, as risk can be evaluated by single value- the loss rate at the occurrence of the unfavorable low-probability event. This brought the VaR measure acceptance almost as standard value, recognized by many researchers. Together with these advantages, application of $V a R$ has disadvantages as well. One of the main drawbacks is absence of subadditivity feature: the sum of $V a R$ measurements of two portfolios can be less, than the risk of value change of the joint portfolio. VaR measurements are also limited to estimating of the marginal loss and do not indicate to other occurrences of possible loss. For eliminating this drawback, Artzner [1] suggested the alternative measurement for market risk evaluation, which meets the subadditivity requirement. They introduced the conditional $\operatorname{VaR}(\mathrm{CVaR})$, called the expected shortfall or the expected tail loss, which indicates the most expected loss of investment, larger than indicated by $V a R$, denoted by conditional expectation:

$$
C V a R=E(R \mid R \geq V a R)
$$

The estimation of both measures of risk evaluation is based on finding adequate quantiles, according to the price distribution data of the analysed financial instrument. The calculated values of $V a R$ and $C V a R$ are more precise, if we have more confident information of the price distribution. Main methods of risk evaluation are based on assumption of normality of return on investment distribution. But empirical research does not confirm the normality of the real data distribution. The shape of profitability distribution has fatter tails, differences in skewness and kurtosis. The fatter tails indicate more often occurrence of extreme unpredictable values, than predicted by the assumption of normality ([3,4,6-9]). The profitability distribution is taller and narrower, than normal distribution. These empirical data indicate that by calculating $V a R$ and $C V a R$ with the assumption of normal distributions, we underestimate real risk value. There are several ways suggested in the research literature to reduce these deviations: substituting normal distribution with the distribution with fatter tails or to use the safety coefficient to compensate inadequacies. The theoretical background of calculating $\operatorname{Var}$ and $C V a R$ risk measures on hourly basis are suggested in the next part. 


\section{Econometric Model for the VaR and CVaR Estimation}

The presented model is based on the mathematical notation, as defined in the RiskMetrics Technical Document [15], and is applied for risk estimation for single type of financial instrument. The input data for suggested model is collected on hourly basis, by registering the opening and closing prices of financial-instrument. Let $P_{o t}$ be the opening price of a given financial instrument at the starting point of hour $t$, and $P_{c t}$ the closing price for the same financial instrument at the end of hour $t$. Then the return $r_{t}$ of one hour period is defined as:

$$
r_{t}=\ln \left(\frac{P_{c t}}{P_{o t}}\right)=\ln \left(P_{c t}\right)-\ln \left(P_{o t}\right)
$$

The model could be defined as adequate, if it could estimate changes of values over time and describe the distribution of return at any point of time. As stated in $[11,15]$, the standard RiskMetrics econometric model meets this requirement only for the estimation of the investment risk for one-day returns analysis, and may give inadequate results while extending or shortening the time period. The market risk at an intraday time horizon has been quantified by Giot P. in [5]. This paper suggests alternative model, where the analysis of returns, based on continuous periods of time, is replaced by the discreet hourly-based return analysis. As the dynamics of the price of a financial instrument is best revealed by the white noise process, the modified model could be based on the following expression:

$$
r_{t}=\mu+\sigma_{t} \cdot \varepsilon_{t}
$$

Here $\mu$ is average alteration of return during the given period of time; $\sigma_{t}$ - standard deviation of return, and $\varepsilon_{t}$ are the independent random values, with the standard normal distribution. Consequently, the return $r_{t}$ has conditional (time-dependent) normal distribution, and the equation (3) can be modified to:

$$
r_{t}=\ln \left(\frac{P_{c t}}{P_{o t}}\right)=\mu+\sigma_{t} \cdot \varepsilon_{t}
$$

According to standard RiskMetrics model $\mu=0$, equation (3) can be simplified to:

$$
r_{t}=\sigma_{t} \cdot \varepsilon_{t}
$$

Return estimations, based on the RiskMetrics model, which assumes normal distribution, slightly differ from those, observed in reality: the tails are fatter, the peak value of the return distribution is higher, and the distribution curve itself is narrower.

In most cases the inadequacies to return distribution are compensated by calculating safety factor or substituting the normal distribution by Student, Laplace, Weibul or distribution mixes [2,11-14]. In the suggested model the risk evaluation will be based on safety factor estimation from the experimental data. 
By using definition (1) it is possible to calculate $\operatorname{VaR}$ as the return $r_{t}$ quantile. While $r_{t}$ is normal distributed with mean $\mu_{t}$ and standard deviation $\sigma_{t}$, the value $z_{t}=\frac{r_{t}-\mu_{t}}{\sigma_{t}}$ will have standard normal distribution. The value of the $5 \%$ quantile is calculated as -1.645 , and the $1 \%$ quantile is 2.326 . Hence:

$$
\begin{aligned}
& P\left(z_{t}<-1.645\right)=P\left(r_{t}<-1.645 \cdot \sigma_{t}\right)=0.05 \\
& P\left(z_{t}<-2.326\right)=P\left(r_{t}<-2.326 \cdot \sigma_{t}\right)=0.01
\end{aligned}
$$

Thus, the 5\% $\operatorname{VaR}$ makes $\operatorname{VaR}_{5 \%}=-1.645 \sigma_{t}$, and the $1 \% \operatorname{VaR}$ makes $\operatorname{VaR}_{1 \%}=-$ $2.326 \sigma_{t}$. For the estimation of the $\operatorname{VaR}, \sigma_{t}{ }^{2}$ must be found out.

$$
\begin{aligned}
& \sigma_{t}^{2}=E\left(r_{t}-E\left(r_{t}\right)\right)^{2}=E\left(r_{t}^{2}-2 \cdot r_{t} \cdot E\left(r_{t}\right)+E\left(r_{t}\right)^{2}=\right. \\
& =E\left(r_{t}^{2}\right)-2 \cdot E\left(r_{t}\right) \cdot E\left(r_{t}\right)+E\left(r_{t}\right)^{2}=E\left(r_{t}^{2}\right)-E\left(r_{t}\right)^{2}
\end{aligned}
$$

According to Phillipe Jorion [10], the first summand of the equation exceeds the impact of the second summand approximately for about 700 times. Therefore:

$$
\sigma_{t}^{2}=E\left(r_{t}^{2}\right)
$$

As the standard RiskMetrics model offers, the $\sigma_{t}^{2}$ is calculated by employing the method of exponential smoothing based on the past data:

$$
\sigma_{t+1}^{2}=\frac{\sum_{i=0}^{\infty} \lambda^{i} \cdot r_{t-i}^{2}}{\sum_{i=0}^{\infty} \lambda^{i}}=(1-\lambda) \cdot \sum_{i=0}^{\infty} \lambda^{i} \cdot r_{t-i}^{2}=(1-\lambda) \cdot r_{t}^{2}+\lambda \cdot \sigma_{t}^{2},
$$

where $0<\lambda<1$. The $C V a R$ is estimated according to the definition (2). As the distribution of $r_{t}$ is standard normal, for each reliability $\mathrm{p}$ we can apply:

$$
\begin{aligned}
& \operatorname{CVaR}_{p}=E\left(r_{t} \mid r_{t} \leq \operatorname{VaR}_{p}\right)=\frac{1}{p \cdot \sigma_{t} \cdot \sqrt{2 \pi}} \int_{-\infty}^{\operatorname{VaR}_{p}} x e^{-\frac{x^{2}}{2 \sigma_{t}^{2}}} d x= \\
& =\left.\frac{\sigma_{t}}{p \cdot \sqrt{2 \pi}} \cdot e^{-\frac{x^{2}}{2 \sigma_{t}^{2}}}\right|_{-\infty} ^{\operatorname{VaR}_{p}}=\frac{\sigma_{t}}{p \cdot \sqrt{2 \pi}} \cdot e^{-\frac{\operatorname{VaR}_{p}{ }^{2}}{2 \sigma_{t}^{2}}}=\frac{e^{-\frac{q_{p}^{2}}{2}}}{p \cdot \sqrt{2 \pi}} \cdot \sigma_{t} .
\end{aligned}
$$

From the formula (4) we can calculate the values of $C V a R_{5 \%}=-2,063 \cdot \sigma_{t}$, and $C V a R_{1 \%}=-2,665 \cdot \sigma_{t}$. In case the return distribution is normal, the evaluations of $V a R$ and $C V a R$ differ only by value of constant: $C V a R_{5 \%}=1,254 \cdot \operatorname{VaR}_{5 \%}$ and $\mathrm{CVaR}_{1 \%}=1,146 \cdot \mathrm{VaR}_{1 \%}$. 


\section{Experimental Verification of the Econometric Model}

For the experimental verification of the suitability of the $V a R$ model we will calculate 5\% VaR and $C V a R$ values for all 24 hours of the day. The long EUR/USD position data was taken from the FOREX (Foreign Exchange Market) currency market reports. The EUR/USD hourly records (total of 6782 records) of opening, closing, min and max prices have been collected during the period since 30 January 2003, 9 p.m. to 2 March 20049 p.m. After sorting data of selected time interval, 219 records were used for the calculation of the $\operatorname{VaR}$ values and the identification of the accuracy of estimation.

The experiment was made in the following steps:

- Verification of the hourly return data fit, under the premise of normal distribution.

- Calculation of the volatility of the trading data, collected under hourly basis.

- $\quad V a R$ and $C V a R$ estimation and analysis.

To verify the data normality, the cumulative function of the observed data distribution was plotted against the theoretical cumulative distribution. The diagrams of P-P plots were slightly higher and narrower than the normal distribution and confirmed the inadequacy of standard model to return distribution, as discussed in the part 2 . The calculation of hourly data volatility showed, that the trade risk increases, when the data volatility is higher. The standard deviations and the data range (max minus min value) of the observed data at the corresponding hours are shown in figure 1 .

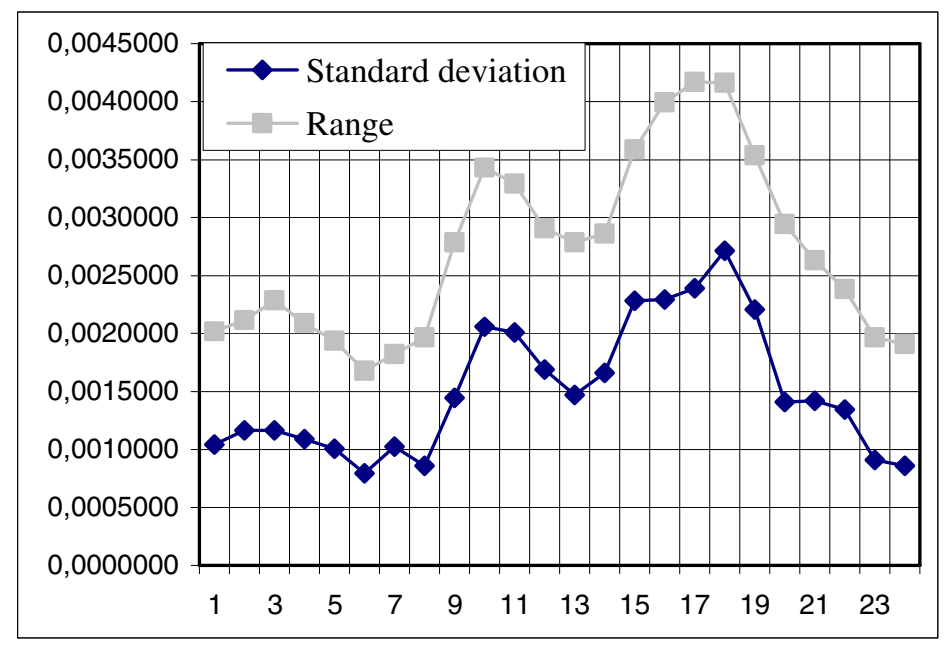

Fig. 1. Standard hourly deviations and range for 24 hours

The experimental calculations pointed out, that the highest volatility of return occured between 2 p.m. and 6 p.m., and the lowest between 10 p.m. and 8 a.m. The biggest observed difference between the highest and the lowest volatility has reached up to $400 \%$. The difference in the volatility allows assuming, that the differences in trading risk could be similar. For calculating $\operatorname{VaR}$ using econometric model, presented in 
the Part 2, the standard return deviation has to be estimated by using exponential smoothing method. All calculations were made with the help of STATISTICA software, Time Series/Forecasting models. The obtained results are presented in Table 1, where $V_{a R_{5}}$ and $C V a R_{5 \%}$ values are estimated for each hour of the day.

Table 1. The VaR and CVaR values for 24 hours

\begin{tabular}{llllll}
\hline Hours & $\mathrm{VaR}_{5 \%}$ & $\mathrm{CVaR}_{5 \%}$ & Hours & $\mathrm{VaR}_{5 \%}$ & $\mathrm{CVaR}_{5 \%}$ \\
$00-01$ & $-0,0014493$ & $-0,001817422$ & $12-13$ & $-0,0020678$ & $-0,002593021$ \\
$01-02$ & $-0,0016142$ & $-0,002024207$ & $13-14$ & $-0,0023456$ & $-0,002941382$ \\
$02-03$ & $-0,0016479$ & $-0,002066467$ & $14-15$ & $-0,0032362$ & $-0,004058195$ \\
$03-04$ & $-0,0015137$ & $-0,001898180$ & $15-16$ & $-0,0032367$ & $-0,004058822$ \\
$04-05$ & $-0,0014080$ & $-0,001765632$ & $16-17$ & $-0,0034310$ & $-0,004302474$ \\
$05-06$ & $-0,0011133$ & $-0,001396078$ & $17-18$ & $-0,0035208$ & $-0,004415083$ \\
$06-07$ & $-0,0014317$ & $-0,001795352$ & $18-19$ & $-0,0027895$ & $-0,003498033$ \\
$07-08$ & $-0,0012031$ & $-0,001508687$ & $19-20$ & $-0,0019280$ & $-0,002417712$ \\
$08-09$ & $-0,0020402$ & $-0,002558411$ & $20-21$ & $-0,0019596$ & $-0,002457338$ \\
$09-10$ & $-0,0028494$ & $-0,003573148$ & $21-22$ & $-0,0018185$ & $-0,002280399$ \\
$10-11$ & $-0,0027626$ & $-0,003464300$ & $22-23$ & $-0,0012586$ & $-0,001578284$ \\
$11-12$ & $-0,0023128$ & $-0,002900251$ & $23-24$ & $-0,0011825$ & $-0,001482855$ \\
\hline
\end{tabular}

Comparing the obtained $\mathrm{VaR}_{5 \%}$ with the characteristics of the hourly data volatility, revealed, that both factors of the market risk estimation possessed a very similar hourly structure, as presented in figure 2 :

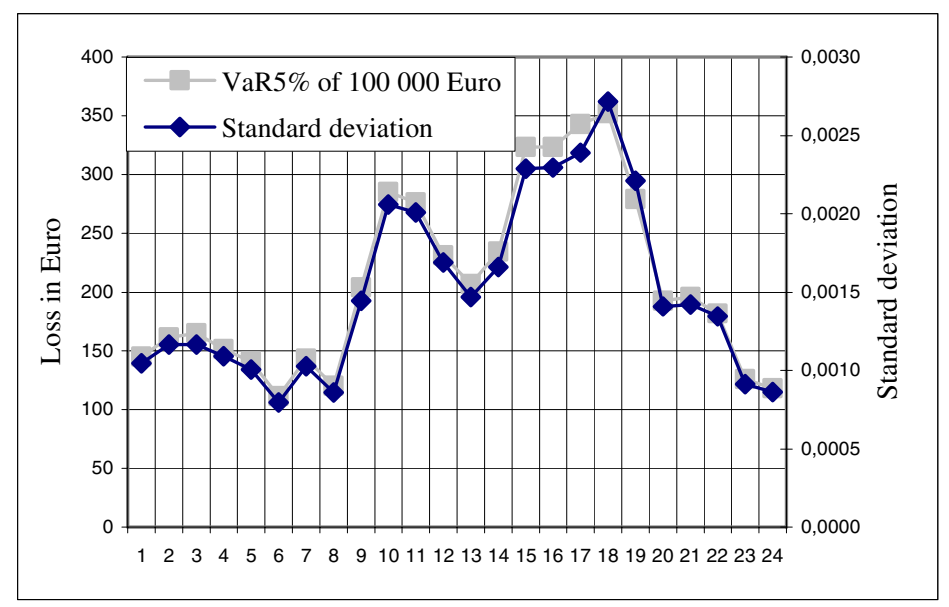

Fig. 2. Plots show the $\mathrm{VaR}_{5 \%}$ estimations and the standard deviation of hourly data

The accuracy of the given econometric model is further defined by finding out, what percent of hourly cycles exceed the estimated VaR. In case the achieved results 
do not considerably differ from $5 \%$, it could be reasonable to rely on the potential results forecasted by the described econometric model. The theoretical and experimental values for the whole hourly cycle are presented in Table 2 .

Table 2. Conformity $\mathrm{VaR}_{5 \%}$ of the theoretical to experimental values

\begin{tabular}{llllll}
\hline Hours & Percent & Hours & Percent & Hours & Percent \\
$00-01$ & 5.47950 & $08-09$ & 6.84930 & $16-17$ & 5.93610 \\
$01-02$ & 4.56621 & $09-10$ & 6.39270 & $17-18$ & 8.21920 \\
$02-03$ & 7.76260 & $10-11$ & 7.30590 & $18-19$ & 6.39270 \\
$03-04$ & 7.30590 & $11-12$ & 7.30590 & $19-20$ & 7.30590 \\
$04-05$ & 4.56620 & $12-13$ & 7.76260 & $20-21$ & 7.30590 \\
$05-06$ & 3.65300 & $13-14$ & 6.84930 & $21-22$ & 6.39270 \\
$06-07$ & 5.93610 & $14-15$ & 5.47950 & $22-23$ & 8.21920 \\
$07-08$ & 5.93610 & $15-16$ & 2.28310 & $23-24$ & 6.39270 \\
\hline
\end{tabular}

The given percent values only slightly exceeded the anticipated 5\% level (the average makes 6.31). The conformity of the model was increased by calculating the safety factor (its estimated value for the experimental data was 1.43), which was used to adjust the values of $V a R$ and $C V a R$ in order to fit the $5 \%$ level:

$$
\operatorname{VaR}_{5 \%}=-1.43 \cdot 1.645 \cdot \sigma_{t} \quad ; C V a R_{5 \%}=-1.43 \cdot 2.063 \cdot \sigma_{t}
$$

According to the estimated hourly values of $\mathrm{VaR}$ or $\mathrm{CVaR}$, the suggested model can help to set more flexible stop-loss rates. The current trading practice with the fixed stop-loss value can lead to substantial loss, where high stop-loss value increases risk to loose big part of investment, and too small value prevents from bigger gains.

The experimental verification of model let us to assume, that together with increasing risk volatility the stop-loss values have to be increased as well. The stop-loss level was evaluated by spread (difference between sell and buy prices), presented in points (1/10 000 change of base currency). By using formulas (5) and the data in Table 1 we can calculate stop-loss boundary values in points. The $\mathrm{VaR}$ or $\mathrm{CVaR}$ values are multiplied by 10,000 , the estimated safety factor 1,43 .

Table 3. Calculated spread for all hours

\begin{tabular}{lllllllll}
\hline & \multicolumn{3}{c}{ Spread } & \multicolumn{3}{c}{ Spread } & \multicolumn{2}{c}{ Spread } \\
Hours & $\mathrm{VaR}_{5 \%}$ & $\mathrm{CVaR}_{5 \%}$ & Hours & $\mathrm{VaR}_{5 \%}$ & $\mathrm{CVaR}_{5 \%}$ & Hours & $\mathrm{VaR}_{5 \%}$ & $\mathrm{CVaR}_{5 \%}$ \\
$00-01$ & 21 & 26 & $08-09$ & 29 & 37 & $16-17$ & 49 & 62 \\
$01-02$ & 23 & 29 & $09-10$ & 41 & 51 & $17-18$ & 50 & 63 \\
$02-03$ & 24 & 30 & $10-11$ & 40 & 50 & $18-19$ & 40 & 50 \\
$03-04$ & 22 & 27 & $11-12$ & 33 & 41 & $19-20$ & 28 & 35 \\
$04-05$ & 20 & 25 & $12-13$ & 30 & 37 & $20-21$ & 28 & 35 \\
$05-06$ & 16 & 20 & $13-14$ & 34 & 42 & $21-22$ & 26 & 33 \\
$06-07$ & 20 & 26 & $14-15$ & 46 & 58 & $22-23$ & 18 & 23 \\
$07-08$ & 17 & 22 & $15-16$ & 46 & 58 & $23-24$ & 17 & 21 \\
\hline
\end{tabular}


In the Table 3 two levels of loss boundary values are presented: for more reserved trading $\left(V a R_{5 \%}\right.$ case) and for the player more tended to risk $\left(C V a R_{5 \%}\right.$ case). It can be stressed, that these coefficients are applied only for EUR/USD position in FOREX.

\section{Conclusions}

This paper suggests the modified RiskMetrics model of risk evaluation for the shortterm investments in currency market. The method is based on calculating VaR and $C V a R$ on hourly basis, using seasonal decomposition. The conformity of the model was increased by calculating the safety factor, which was used to adjust the values of VaR and $C V a R$. The experimental verification of model showed that together with increasing risk volatility the stop-loss values have to be increased as well. The main results presented in the article provide basis for further research by applying the suggested econometric model for risk evaluation of short-time investment in the currency market.

\section{References}

1. Artzner, P., Delbaen, F., Eber, J., Heath, D.: Coherent Measures of Risk. Mathematical Finance, Vol. 9(3). Backwell publishers, Malden USA (1999) 203-228

2. Benninga, S., Wiener, Z.: Value-At-Risk (Var). Mathematica in Education and Research, Vol. 7(4). Springer Verlag, New York (1998) 39-45

3. Carr, P., Geman, H., Madan, D.B., Yor, M.: The Fine Structure of Asset Returns: an Empirical Investigation. Journal of Business, No.75. The University of Chicago Press (2002) 305-332

4. Christoffersen, P., Diebold F.: How Relevant Is Volatility Forecasting For Financial Risk Management? Review Of Economics and Statistics, Vol. 82. MIT Press, Massachusetts USA (2000) 1-11

5. Giot, P.: Market risk models for intraday data. European Journal of Finance, Vol. 11(4). Routledge (Taylor \& Francis), UK (2005) 309-324

6. Gopikrishnan, P., Meyer, M., Amaral, L.A.N., Stanley, H.E.: Inverse Cubic Law for the Distribution of Stock Price Variations. European Physical Journal, Springer-Verlag, Berlin Heidelberg New York (1998) 139-140

7. Danielsson, J., de Vries, C.: Value-at-Risk and Extreme Returns. Annales d'Economie et Statistique, Centre d'études de l'emploi Vol. 3 (2000) 73-85

8. Dowd, K.: Measuring Market Risk. John Wiley \& Sons USA (2002)

9. Franke, J., Hardle, W., Stahl, G.: Measuring Risk in Complex Stochastic Systems. Springer-Verlag, Berlin Heidelberg New York (2000)

10. Jorion, P.: Value at Risk: the New Benchmark for Managing Financial Risk. McGraw-Hill (2000)

11. 11. Silvapulle, P., Granger, C.W.J.: Large Returns, Conditional Correlation and Portfolio Diversification: a Value-at-Risk Approach. Quantitative Finance, Vol.1(5). Routledge (Taylor \& Francis), UK (2001) 542-551

12. Shapiro, S.S., Wilk, M.B., Chen, H.J.: A Comparative Study of Various Tests of Normality. Journal of the American Statistical Association, Vol 63. ASA, Boston (1968) 1343-1372

13. Rachev, S., Mittnik, S.: Stable Paretian Models in Finance. John Wiley \& Sons (2000)

14. Randal, J.A., Thomson, P.J., Lally, M.T.: Non-Parametric Estimation of Historical Volatility. Quantitative Finance, Vol 4(4). Routledge (Taylor \& Francis), UK (2004) 427-440

15. Riskmetrics Technical Document. 4th edn. J.P. Morgan/Reuters, New York (1996) 